\title{
Stabilization of a stimulated Brillouin fiber ring laser by strong pump modulation
}

\author{
Jean Botineau, Claude Leycuras, Carlos Montes, and Eric Picholle \\ Laboratoire de Physique de la Matière Condensée, Université de Nice, Parc Valrose, 06034 Nice Cédex, France
}

Received March 1, 1988; accepted November 17, 1988

\begin{abstract}
A stable train of compressed Stokes pulses (to $\sim 10 \mathrm{nsec}$ ) is obtained in a stimulated Brillouin fiber ring laser (of length $L=83 \mathrm{~m}$ ) by periodically interrupting the argon-ion cw pump beam with an intraring cavity acousto-optic modulator. Interruption of the pump action, at each round-trip time $t_{r} \simeq L n / c$, permits damping of the excited sound waves that accumulate at the entry of the fiber owing to the inertial response of the material, well described by the coherent three-wave stimulated Brillouin scattering model (C3W-SBS equations). Amplification and compression of the backscattered Stokes pulse are limited by nonlinear optical Kerr effect, which is incorporated into the C3W-SBS equations.
\end{abstract}

\section{INTRODUCTION}

Stimulated Brillouin backscattering (SBS) is a dominant nonlinear effect in single-mode optical fibers, where it appears at moderate threshold values. ${ }^{1,2}$ In particular, SBS is used as a gain process in fiber lasers, ${ }^{3-8}$ in which a narrowlinewidth laser pump source, yielding high-power flux densities into the fiber, amplifies the stimulated backscattered Stokes background on a long interaction length. Generation of periodic trains of short Brillouin Stokes pulses has been attributed to mode locking, $, 5,9,10$ i.e., to phase synchronization of longitudinal modes in the first Stokes component that cover the Brillouin gain bandwidth. Mode locking may be installed by the relaxation behavior that occurs in the oscillators as a result of periodic pump depletion or active modulation. However, the temporal behavior of the SBS process describing these finite-cell-length oscillations ${ }^{11}$ or relaxation oscillations ${ }^{12}$ has been investigated within the strongly damped approximation (called here the SDA model) for the sound wave, which is relevant only for the stationary case ${ }^{13}$ or for weak Stokes pulses, as we shall discuss below. For long interaction times and high amplification and compression of the Stokes pulse a description starting from the well-established coherent three-wave SBS equations derived for electrostrictive continuous media, ${ }^{14,15}$ called here C3W-SBS equations, is necessary (note that the resonant condition in this model implies second-order nonlinearities); the asymptotic behavior of an initial bounded Stokes pulse whose amplitude grows and whose width shrinks at the expense of a continuous pump wave has been studied in Refs. 16 and 17.

Our aim in this paper is to compare experimental results obtained in fiber ring configurations with those provided by numerical simulation of the appropriate C3W-SBS equations through the slowly varying amplitude approximation, which is valid here for the optical waves as well as for the sound wave. Modeling was done simultaneously with an experiment on a single-mode silica-fiber ring laser, of length $L=83 \mathrm{~m}$, pumped by a single-mode argon-ion cw laser. The properties determined from the numerical simulation have permitted improvement of the experimental configuration.
For example, numerical calculations show that, when the backscattered Stokes pulse leaves the nonlinear medium and $\mathrm{cw}$ pump input is maintained, the amplitude of the sound wave can remain high, especially at the fiber end used for the pump coupling. This accumulation of the excited phonons owing to the inertial response of the sound waves, well described by the C3W-SBS equations, is responsible for shortening the effective length of the nonlinear medium by depleting the pump just at its entry and for spreading the Stokes-pulse tail. Thus one of the aims of an improved experimental setup is to permit spontaneous phonon damping by interrupting the pump input coupling into the fiber at a frequency equal to the round-trip photon frequency $\left(\nu_{r}=\right.$ $\left.1 / t_{r} \simeq c / L n\right)$ with an acousto-optic modulator (AOM) in order to stabilize the amplified and compressed train of Brillouin Stokes pulses. This device also prevents reflections of the Stokes pulses on the output laser pump mirror by isolating the laser pump from the backscattered Stokes pulses. We improve our description of the finite spatial width of the Stokes pulses observed in the actual experiment by including optical Kerr nonlinearity in the C3W-SBS equations, which introduces phase modulation, ${ }^{18}$ and here amplitude modulation too, and limits the compression.

C3W-SBS equations have already been used to interpret pulse compression (from 20 to 2 nsec) by backward SBS in a tapered optical waveguide filled with methane at $130 \mathrm{~atm} .{ }^{19}$ However, in nonlinear-optical problems dealing with SBS (and also with stimulated Raman scattering), the SDA model is commonly used for the dynamical equations. ${ }^{11-13}$ This model may be derived from C3W-SBS equations by assuming that the sound response is instantaneous and the phonons are motionless. Such a description reduces the system to the evolution of two counterpropagating optical waves coupled together by third-order susceptibility: it amounts to considering two equations involving optical intensities, disregarding phases. The SDA model is exactly solvable ${ }^{20}$ but obviously cannot describe the interaction outside the spatial overlapping domain of the two electromagnetic wave envelopes.

In Section 2 we recall the coherent SBS interaction briefly, and we present the basic equations that are used for the 
numerical simulations. In Section 3 we describe the experimental setups and give the experimental results for the two ring configurations used as fiber oscillators. The first simple setup, without an AOM, yields a nonstable regime, and we observe that when pump intensity is increased well above SBS threshold several Stokes pulses are simultaneously excited along the fiber, among which are stationarily amplified only those that are separated by submultiples of the roundtrip period. To explain this fact we present a simple argument based on stationarity, total pump depletion by each leading Stokes pulse, and exponential SBS Stokes gain. The second setup, in which the AOM is tuned to the roundtrip photon frequency $\nu_{r}$, couples the laser input to the fiber ring oscillator through first-order Bragg diffraction, while feedback is achieved through zero-order Bragg diffraction. This setup yields a stable train of amplified and compressed Brillouin Stokes pulses. In Section 4 we give the numerical results: the time behavior of the field amplitudes governed by C3W-SBS plus the optical Kerr equations agrees well with the experimental results. Finally, a conclusion is drawn in Section 5 in which the possibility of quasi-solitons in the C3W-SBS dynamics is envisaged.

\section{MODELS OF STIMULATED BRILLOUIN SCATTERING}

\section{A. Coherent Three-Wave SBS Model}

In SBS a forward-propagating pump wave (at frequency $\omega_{1}$ $=k_{1} c$ ) couples with the thermal phonon fluctuations of the medium and stimulates a counterpropagating Stokes beam (at frequency $\omega_{2}=\omega_{1}-\omega_{s}$ ), downshifted by the phonon frequency $\omega_{s}=2 c_{s} \omega_{1} n / c$, where $n$ is the refractive index, $c_{s}$ the sound velocity, and $c$ the velocity of light in vacuum. The resonance condition for three-wave coherent interaction provides maximum power transfer when the wave-vector mismatch is zero: $\mathbf{k}_{s}=\mathbf{k}_{1}-\mathbf{k}_{2}$. In a single-mode fiber it is possible to reduce the wave description to three planar homogeneous propagating waves by following a treatment similar to that of Ref. 21: (1) a forward-propagating pump wave $\tilde{E}_{1}(x, t)=E_{1}(x, t) \exp \left[i\left(k_{1} x-\omega_{1} t\right)\right]$ exhibiting depletion during the amplification and compression of (2) a backscattered Stokes wave $\tilde{E}_{2}(x, t)=E_{2}(x, t) \exp \left[-i\left(k_{2} x+\omega_{2} t\right)\right]$ and (3) a forward-damped sound wave $\tilde{\rho}_{s}(x, t)=\rho_{s}(x, t) \exp \left[i\left(k_{s} x\right.\right.$ - $\left.\omega_{s} t\right)$ ] induced by electrostriction. Counterpropagation then yields the phase-matching condition: $k_{s}=k_{1}+k_{2} \simeq$ $2 k_{1}$.

To describe the coherent time-dependent behavior of SBS in single-mode fibers, we can use the well-established C3WSBS equations derived for electrostrictive continuous media, ${ }^{14,15}$ which concern the three coupled waves, $\tilde{E}_{1}, \tilde{E}_{2}$, and $\tilde{\rho}_{s}$, having amplitudes $E_{1}, E_{2}$, and $\rho_{s}$, respectively, and some damping $\gamma_{i}$. In one-dimensional optical media of dielectric permeability $\epsilon$, and for parallel polarized electromagnetic waves, we can write

$$
\begin{aligned}
{\left[\partial_{t}^{2}+2 \gamma_{1} \partial_{t}-(c / n)^{2} \partial_{x}^{2}\right] \tilde{E}_{1} } & =-\left(1 / n^{2}\right) \partial_{t}^{2}\left[(\partial \epsilon / \partial \rho) \tilde{\rho}_{s} \tilde{E}_{2}\right] \\
{\left[\partial_{t}^{2}+2 \gamma_{2} \partial_{t}-(c / n)^{2} \partial_{x}^{2}\right] \tilde{E}_{2} } & =-\left(1 / n^{2}\right) \partial_{t}^{2}\left[(\partial \epsilon / \partial \rho) \tilde{\rho}_{s}^{*} \tilde{E}_{1}\right] \\
\left(\partial_{t}^{2}+2 \gamma_{s} \partial_{t}-c_{s}{ }^{2} \partial_{x}^{2}\right) \tilde{\rho}_{s} & =-\left(\rho_{0} \epsilon_{0} / 2\right)(\partial \epsilon / \partial \rho) \partial_{x}{ }^{2}\left(\tilde{E}_{1} E_{2}^{*}\right)
\end{aligned}
$$

It is usual to introduce the slowly varying envelope approximation for the waves; the complex amplitudes $E_{1}, E_{2}$, and $\rho_{s}$ are assumed slowly varying in time and space so that the development of the second derivatives of the complete fields $\left(\tilde{E}_{1}, \tilde{E}_{2}\right.$, and $\left.\tilde{\rho}_{s}\right)$ yields

$$
\begin{aligned}
& \partial_{t}{ }^{2} \rightarrow-2 i \omega_{j} \partial_{t}+\partial_{t}{ }^{2}-\omega_{j}^{2} \simeq-2 i \omega_{j} \partial_{t}-\omega_{j}{ }^{2}, \\
& \partial_{x}{ }^{2} \rightarrow \pm 2 i k_{j} \partial_{x}+\partial_{x}{ }^{2}-k_{j}^{2} \simeq \pm 2 i k_{j} \partial_{x}-k_{j}{ }^{2},
\end{aligned}
$$

where the right-hand operators apply only to the amplitudes. This is a good approximation for the electromagnetic waves since their frequencies $\omega_{j}$ are high compared with the time variation $\partial_{t}$ of the amplitude. For the sound waves two cases are possible: (1) In the strong-coupling case $\left(\Phi>\Phi_{c}=\right.$ $6 \times 10^{12} \mathrm{~W} / \mathrm{cm}^{2}$ in silica) the sound response time $\omega_{s}{ }^{-1}$ may be greater than the inertial response time $\tau=\left(\partial_{t} \rho_{s} / \rho_{s}\right)^{-1}$, and the dynamics associated with the second derivative must be included, which leads to a complex phase-amplitude evolution $^{17}$ (this case is relevant for laser-plasma interaction problems). (2) However, in typical optical-fiber experiments we are addressing the small-coupling case $\left(\Phi \ll \Phi_{c}\right)$, and the slowly varying envelope approximation for the sound-wave amplitude evolution holds, too. ${ }^{16}$ The electrostrictive field $E_{s}$ associated to the phonon amplitude $\rho_{s}$ is given by $\rho_{s}=i \sigma E_{s}$, where $\sigma=\left(\rho_{0} n^{3} \epsilon_{0} / 2 c c_{s}\right)^{1 / 2}$ and $\epsilon_{0}$ is the vacuum dielectric constant. We write Eqs. (1)-(3) in dimensionless form after introducing time $\left(\tau=1 / K E_{p}\right)$ and length $(\Lambda=c \tau / n)$ scales, where $E_{p}$ is the input amplitude of the pump $E_{1}$ and

$$
K=\left(\frac{\epsilon_{0} c n^{7}}{2 \rho_{0} c_{s}}\right)^{1 / 2} \frac{\pi p_{12}}{\lambda}
$$

is the SBS coupling constant for optical materials ${ }^{2}$; for an optical fiber of fused silica with a refractive index $n=1.46$, an elasto-optic coefficient $p_{12}=0.286$, a sound velocity $c_{s}=$ $5.96 \times 10^{3} \mathrm{~m} \mathrm{sec}^{-1}$, and an unperturbed fiber density $\rho_{0}=$ $2.21 \times 10^{3} \mathrm{~kg} \mathrm{~m}^{-3}$, we obtain for the pump wavelength $\lambda=$ $5145 \AA$ the SBS coupling value $K=65.9 \mathrm{~m} \mathrm{sec}^{-1} \mathrm{~V}^{-1}$. If we change $\left(E_{i} / E_{p} \rightarrow E_{i} ; t / \tau=t K E_{p} \rightarrow t ; x / \Lambda=x n K E_{p} / c \rightarrow x\right)$, introduce $\left(\mu=\gamma_{s} \tau=\gamma_{s} / K E_{p} ; \mu_{e}=\gamma_{1,2} \tau=\gamma_{1,2} / K E_{p}\right)$, and neglect the sound-wave velocity in Eq. (3) (because $n c_{s} / c \sim$ $10^{-5}$ in silica), Eqs. (1)-(3) yield the dimensionless form

$$
\begin{aligned}
\left(\partial_{t}+\partial_{x}+\mu_{e}\right) E_{1} & =-E_{2} E_{s}, \\
\left(\partial_{t}-\partial_{x}+\mu_{e}\right) E_{2} & =E_{1} E_{s}^{*}, \\
\left(\partial_{t}+\mu\right) E_{s} & =E_{1} E_{2}^{*} .
\end{aligned}
$$

Starting from an initial bounded Stokes background $E_{2}$, the transient and asymptotic evolution governed by Eqs. (5)-(7) in the presence of a constant input pump wave $E_{1}$ without optical attenuation ( $\left.\mu_{e}=0\right)$ has been considered in Ref. 16 . During the first stage, the Stokes-pulse growth is exponential until its amplitude becomes comparable with the pump amplitude. A second regime follows in which total and abrupt depletion of the pump field $E_{1}$ is accompanied by a sharp leading edge of the backscattered Stokes envelope $E_{2}$, which is amplified and compressed. The backscattered wave envelope exhibits a set of large peaks of decreasing amplitude, the amplitude of the first growing linearly with time $t$ while its width shrinks as $1 / t$. Later there is a spatial spreading of the Stokes tail because of the finite response time of the sound waves (inertial response time). The 
phases of the complex amplitudes $E_{i}$ are time and space independent, except for the sudden $\pi$ shifts that appear when the fields vanish ( $\pi$ pulse envelope ${ }^{22}$ ).

\section{B. SBS Adiabatic Model: SDA Model}

In order to compare the C3W-SBS model governed by Eqs. (5) - (7) with the commonly used adiabatic model ${ }^{11,12,13,20}$ in which the inertial response of the sound wave is neglected $\left(\mu E_{s} \gg \partial_{t} E_{s}\right)$, let us write the evolution equations for the flux intensities $\Phi_{i}=\left(n \epsilon_{0} c / 2\right)\left|E_{i}\right|^{2}$ by setting $E_{s}=E_{1} E_{2}{ }^{*} / \mu$ in Eq. (7) in dimensioned units:

$$
\begin{aligned}
& {\left[\partial_{x}+(n / c)\left(\partial_{t}+\gamma_{e}\right)\right] \Phi_{1}=-g \Phi_{1} \Phi_{2},} \\
& {\left[\partial_{x}-(n / c)\left(\partial_{t}+\gamma_{e}\right)\right] \Phi_{2}=-g \Phi_{1} \Phi_{2}}
\end{aligned}
$$

where the Brillouin gain coefficient $g$ is given by

$$
g=\frac{4}{\gamma_{s} \epsilon_{0} c^{2}} K^{2}=\frac{2 \pi n^{7} p_{12}{ }^{2}}{c \lambda^{2} \rho_{0} c_{s} \Delta \nu_{B}}=4.62 \times 10^{-11} \mathrm{~m} \mathrm{~W}^{-1},
$$

where $\Delta \nu_{B}=150 \mathrm{MHz}$ is the Brillouin linewidth (FWHM) in pure fused silica. ${ }^{23,24}$

If we consider the amplification regime already described for an initial spatial bounded Stokes envelope $E_{2}$ in the presence of a constant pump $E_{1}$, the SDA model (which is exactly solvable ${ }^{20}$ ) properly describes the first interaction stage where $E_{2}$ exhibits exponential amplification and steepening (compression) during its backward motion at the expense of $E_{1}$, which is depleted. ${ }^{16}$ When $E_{2}$ is greater than $E_{1}$ and the width of the $E_{2}$ peak becomes extremely small, the characteristic evolution time becomes smaller than the damping time $\gamma_{s}^{-1}$ of the sound wave $\left(\partial_{t} \rho_{s} \gg \gamma_{s} \rho_{s}\right.$, i.e., $\partial_{t} E_{s} \gg$ $\left.\mu E_{s}\right)$, the SDA adiabatic model fails. Neither the linear amplification with time of the leading $E_{2}$ peak, obtained with the C3W-SBS equations, nor the spreading of its tail is described through the SDA model; indeed, that model cannot account for the interaction between the sound wave and the pump wave after (or outside) the Stokes-pump interaction, because within this approximation the sound wave vanishes in the absence of $E_{1}$ or $E_{2}$. In particular, the SDA model will be inappropriate to describe pump-wave-Stokes- pulse interactions in a ring configuration (such that considered here), in which the active medium retains a memory of former interactions, because of the finite response time of sound waves.

\section{SBS Coupled to the Nonlinear-Optical Kerr Effect}

When the SBS amplification and compression of the backscattered Stokes pulse becomes important we must look for saturation mechanisms in order to interpret the finite spatial width observed in the actual experiment. For a time width of the order of $\gamma_{s}^{-1}$, i.e., for few nanoseconds, the dispersion does not act efficiently. However, self-phase modulation due to the nonlinear-optical Kerr effect ${ }^{18}$ seems to be a good candidate because it couples phases and amplitudes, producing self-amplitude modulation and spreading the Stokes pulse, like the time behavior obtained for a similar problem in which dispersion instead of the SBS effect is taken into account. ${ }^{25,26}$ The refractive index of the fiber medium is

$$
n=n_{0}+n_{2}|\tilde{E}|^{2} / 2 \text {, }
$$

where $n_{0}(=1.46)$ is the unperturbed refractive index, $n_{2}$ $\left(=1.27 \times 10^{-22} \mathrm{~m}^{2} \mathrm{~V}^{-2}\right.$ for silica $\left.{ }^{18}\right)$ is the Kerr coefficient, and $\tilde{E}=\tilde{E}_{1}+\tilde{E}_{2}$ is the total electromagnetic field. Here, we retain only the zero-frequency terms of the nonlinear Kerr effect, which describe a uniform bias of the nonlinear index. The other terms, called holographic terms, ${ }^{27}$ are oscillating terms at frequencies different from those involved in SBS, namely, at $\exp [3 i(k x \pm \omega t)], \exp [i(3 k x \pm \omega t)]$, and $\exp [i(k x \pm$ $3 \omega t)]$. They can in themselves be responsible for Raman instabilities ${ }^{27}$ but with lower gains than the SBS process considered here. Nevertheless, the bias terms of the refractive index are resonant with SBS and will be responsible for self-phase and self-amplitude modulation, which considerably modify the amplitude profile and width of the backscattered amplified Stokes pulse. Introducing the nonlinear optical Kerr effect into the dimensionless C3W-SBS Eqs. (5)-(7), we obtain the dimensionless C3W-SBS-K equations

\begin{tabular}{|c|c|c|c|c|c|}
\hline \multirow[b]{2}{*}{ Other Parameters } & \multicolumn{5}{|c|}{$P(\mathrm{~mW})$} \\
\hline & 25 & 50 & 100 & 150 & 200 \\
\hline$\Phi\left(\mathrm{MW} / \mathrm{cm}^{2}\right)$ & 0.347 & 0.694 & 1.38 & 2.08 & 2.77 \\
\hline$E_{p}(\mathrm{MV} / \mathrm{m})$ & 1.35 & 1.91 & 2.70 & 3.30 & 3.82 \\
\hline$\tau=\left(K E_{p}\right)^{-1}$ (nsec) & 11.2 & 7.94 & 5.62 & 4.60 & 3.97 \\
\hline$\Lambda=c \tau / n(\mathrm{~m})$ & 2.30 & 1.63 & 1.15 & 0.945 & 0.815 \\
\hline$L / \Lambda$ & 36 & 51 & 72 & 88 & 101 \\
\hline$\mu=\gamma_{s} \tau$ & 5.26 & 3.73 & 2.64 & 2.16 & 1.86 \\
\hline$\mu_{e}=\gamma_{e} \tau$ & $5.7 \times 10^{-3}$ & $4.0 \times 10^{-3}$ & $2.9 \times 10^{-3}$ & $2.3 \times 10^{-3}$ & $2.0 \times 10^{-3}$ \\
\hline$K_{r}=n_{2} \omega E_{p} / 2 K$ & $4.6 \times 10^{-3}$ & $6.5 \times 10^{-3}$ & $9.1 \times 10^{-3}$ & $1.11 \times 10^{-2}$ & $1.29 \times 10^{-2}$ \\
\hline
\end{tabular}

$$
\left(\partial_{t}+\partial_{x}+\mu_{e}\right) E_{1}=-E_{2} E_{s}+i K_{r}\left(\left|E_{1}\right|^{2}+2\left|E_{2}\right|^{2}\right) E_{1},
$$

Table 1. Computation Parameters for the Fiber Used in the Experiments ${ }^{a}$

${ }^{a}$ Length $L=83 \mathrm{~m}$, effective cross section $S=7.2 \times 10^{-12} \mathrm{~m}^{2}$. Other notation:

$P$ is the pump power coupled into the fiber

$\Phi$ is the pump flux intensity

$E_{p}$ is the electromagnetic field corresponding to $\Phi$

$\tau$ is the SBS characteristic time [where the coupling constant given by Eq. (4) is $K=65.9 \mathrm{~m} \mathrm{sec}^{-1} \mathrm{~V}^{-1}$ ]

$\Lambda$ is the SBS characteristic length

$\mu$ is the dimensionless sound-damping rate (corresponding to $\gamma_{s}=\pi \Delta \nu_{B}=470 \mathrm{MHz}$ )

$\mu_{e}$ is the dimensionless optical attenuation (corresponding to $21.7 \mathrm{~dB} \mathrm{~km}^{-1}$ )

$\mathrm{K}_{r}$ is the dimensionless Kerr/SBS rate coefficient (where $n_{2}=1.27 \times 10^{-22} \mathrm{~m}^{2} \mathrm{~V}^{-2}$ )

$\omega$ is the laser frequency $\left(=3.66 \times 10^{15} \mathrm{sec}^{-1}\right)$. 


$$
\begin{aligned}
\left(\partial_{t}-\partial_{x}+\mu_{e}\right) E_{2} & =E_{1} E_{s}{ }^{*}+i K_{r}\left(2\left|E_{1}\right|^{2}+\left|E_{2}\right|^{2}\right) E_{2}, \\
\left(\partial_{t}+\mu\right) E_{s} & =E_{1} E_{2}{ }^{*},
\end{aligned}
$$

where the dimensionless coefficient comparing the nonlinear-optical Kerr effect with the SBS effect is given by

$$
K_{r}=n_{2} \omega E_{p} / 2 K,
$$

$K\left(\mathrm{~m} \mathrm{sec}^{-1} \mathrm{~V}^{-1}\right)$ being the SBS coupling coefficient given by Eq. (4).

The validity domain of Eqs. (11) and (12) is restricted to the perturbative Kerr contribution. For the pump intensities used here, we have $K_{r} \ll 1$ (see Table 1 ), which gives an upper limit for the amplified $E_{2}$ field $\left(E_{2}<13\right.$ in the $E_{p}$ units used here) within this description. Redimensionalization of Eqs. (11) and (12), by including $K_{r}$ in new field, time, and space variables, permits suppression of $K_{r}$ from these equations with the validity condition reduced to new field amplitudes smaller than unity.

Within the pure C3W-SBS description given by Eqs. (5)(7), the simple phase dynamics already discussed reduces the problem to a study of three partial differential equations for the real part of $E_{i}{ }^{16}$ With the SBS-K Eqs. (11), (12), and (7) the phase dynamics plays a nontrivial role: the nonlinear coupled phase-amplitude evolution leads to amplitude modulation and subsequently spreads the width of the amplified $E_{2}$ pulse.

\section{EXPERIMENTAL PROCEDURE AND RESULTS}

The purpose of the experiment described here is to design a proper setup for SBS analysis in a planar-wave configuration. The active medium is an 83-m-long single-mode rectilinearly birefringent fiber $\mathrm{F}$, with a. 3- $\mu \mathrm{m}$-diameter puresilica core surrounded by a borosilicate optical cladding, used along one of its neutral axes, such that the interacting optical waves are parallel polarized. In order to have a large interaction length, we pump the fiber with a cw single-mode ionized argon-ion laser emitting at $\lambda=5145 \AA$. To work with low pumping power levels, we mount the fiber in an optical ring oscillator in which the feedback is achieved by the two mirrors, $\mathrm{M}_{1}$ and $\mathrm{M}_{2}$; input and output fiber couplings are obtained through $18 \times$ microscope lenses (see Figs. 1 and 2). Detection is achieved with a 500-psec rise-time silicon diode connected to a $400-\mathrm{MHz}$ oscilloscope. This mounting set then acts as an optically pumped Brillouin fiber laser. ${ }^{3-9}$ We define the forward direction in the ring oscillator as the pump-beam direction.

Thermal phonon fluctuations in the fiber are then amplified by the cw pump beam to give rise to backward-scattered Brillouin Stokes pulses, which are recoupled into the fiber after a round trip in the oscillator for further amplification. The pump wave's coupling efficiency is $60 \%$, and the fiber's linear attenuation is $34 \%$. We estimate the input coupling efficiency of the Brillouin pulses to be $80 \%$ and the output fiber coupling efficiency to be $90 \%$, taking lens losses into account.

A ring oscillator configuration ${ }^{3,4,9}$ rather than a back-andforth oscillator ${ }^{5,6,28}$ was chosen in order to optimize the interaction length. We have successively used the two ring oscillators shown in Figs. 1 and 2 to solve different experimental problems described below.

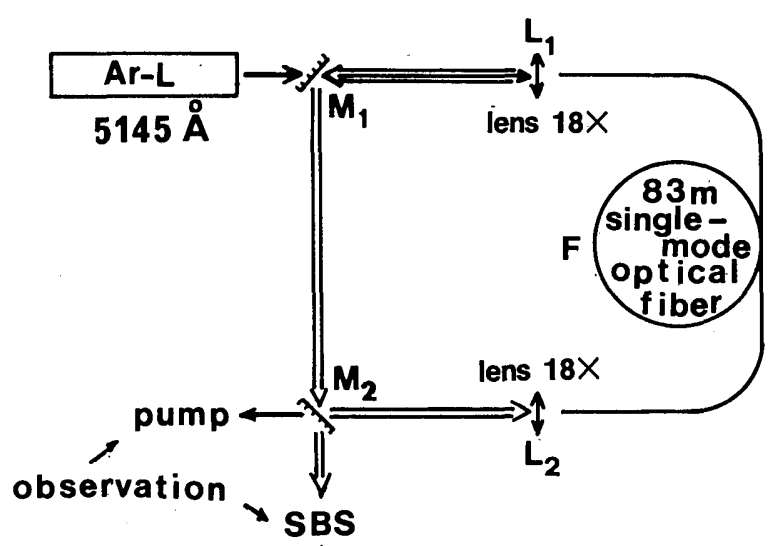

Fig. 1. Basic mounting set of the first stimulated Brillouin ring fiber oscillator: constant pump input.

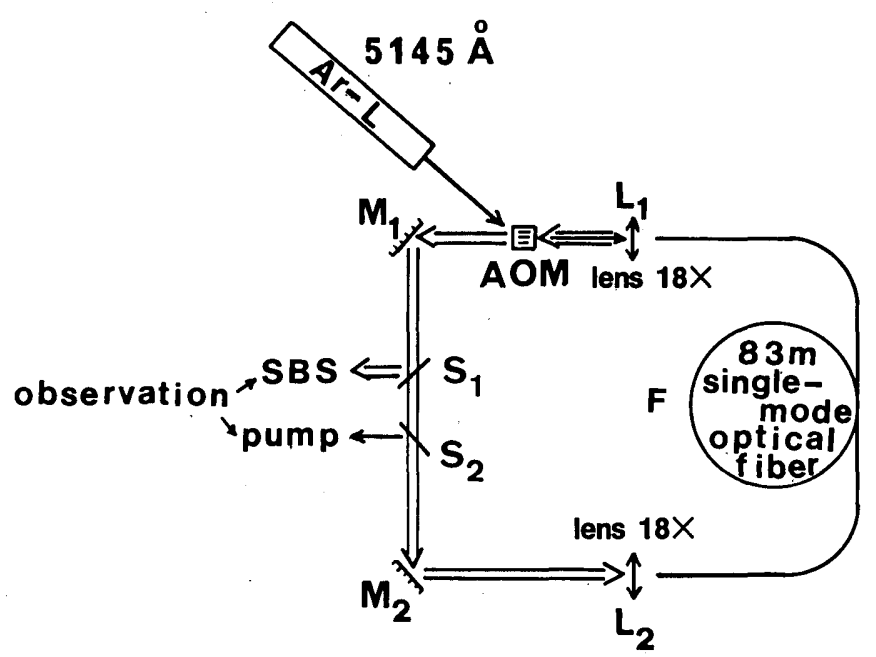

Fig. 2. Mounting set for the second stimulated Brillouin ring fiber oscillator: modulated pump input.

\section{A. First Ring Oscillator}

We start with the simplest configuration, the experimental setup of which is depicted in Fig. 1. The two mirrors $M_{1}$ and $\mathrm{M}_{2}$ are $50 \%$ beam splitters. Pump coupling is achieved through $\mathrm{M}_{1}$, and either the output pump beam or the Brillouin pulses may be observed through $\mathrm{M}_{2}$.

\section{Experimental Results}

The backward output signal is recorded with a fast detector, while the laser pump power level is progressively increased to $600 \mathrm{~mW}$. At low pump level, backward scattering originates mainly in cw Rayleigh scattering in the fiber and from reflections upon the different optical surfaces. When the pump power reaches a characteristic threshold value (here less than $50 \mathrm{~mW}$ at the output of the pump argon laser), a temporal structure arises in the backward beam, consisting of a periodic train of stimulated Brillouin pulses, the temporal repetition rate of which corresponds to a pulse round-trip time in the ring $t_{r}=1 / \nu_{r}(=415 \mathrm{nsec}$ within this configuration, taking into account the 11-nsec transit time outside the fiber). The temporal width of the Brillouin pulses is typically in the 20-30-nsec range, with a sharp peak, the maximum of which is difficult to determine but may be estimated as being several times the pump power coupled into the fiber 


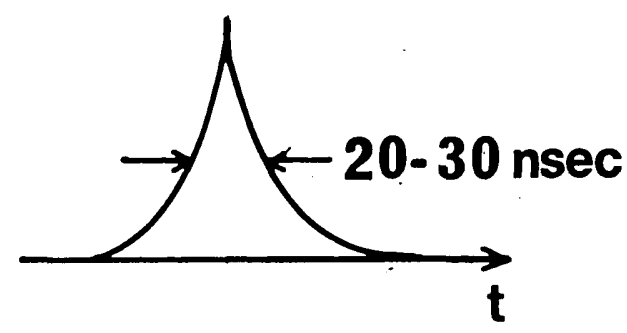

a

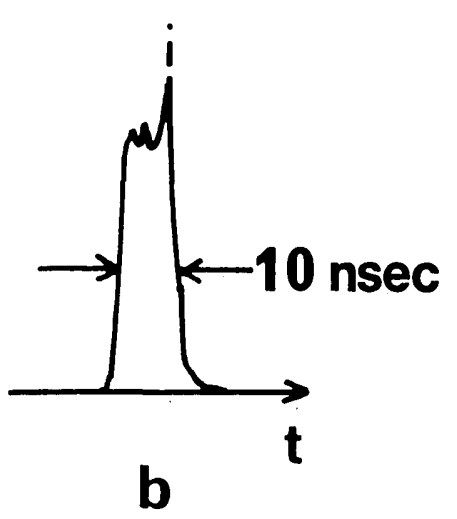

Fig. 3. Typical stimulated Brillouin pulses obtained at the output of the oscillator of Fig. 1.

(Fig. 3a). In fact, this amplitude is highly unstable and fluctuates between zero and a maximum value; sometimes some sharper and narrower pulses appear, probably with an internal fine structure not resolved by our detector (Fig. $3 \mathrm{~b}$ ).

We also measure the average output pump power level, which exhibits a saturation value close to $30 \mathrm{~mW}$ directly after the fiber and half of this value at the oscillator output. On the other hand, the average backward-scattered power is almost linear with the input power.

Another interesting feature is the pulse-repetition-rate evolution with the pump power level. As we pointed out in Section 1 and has been noted by several authors, ${ }^{28,29}$ when stimulation occurs the frequency repetition rate of the Brillouin pulses, corresponding to a pulse round-trip time $t_{r}$ $(=415 \pm 5 \mathrm{nsec})$ at the low pump level, doubles at the higher pump level (210 nsec between two pulses). When the pump power level is increased still more, we observe that the repetition rate is multiplied by three and then by four and even five, with an increasing instability among all these regimes. At such a pump power level (some hundreds of milliwatts at the output of the argon laser) high transient regimes of randomly distributed Brillouin pulses appear. When the repetition rate increases by an integer factor $(2,3, \ldots)$, the pulse width shown in Fig. 3 a seems to decrease in the same ratio.

We also have successively probed the backward- and forward-output frequency power spectra, using the experimental setup of Figs. 4 and 5 . These experimental mountings use a 165-mm Jobin \& Yvon holographic diffraction grating engraved with 1872 lines/mm and a $50-\mathrm{cm}$ focal-length and f/9 aperture objective well corrected for aberrations (Clairaut objective). This setup gives a theoretical spectral resolution of less than $2 \mathrm{GHz}$, which ensures a good separation for the Brillouin lines $\left(\nu_{s}=34 \mathrm{GHz}\right)$ but does not give any information on the fine structure of each individual line $\left(\delta \nu_{2}\right.$ $<\Delta \nu_{B}=150 \mathrm{MHz}$, where $\delta \nu_{2}$ corresponds to the shortest measured time width $\Delta t_{2}=10 \mathrm{nsec}$ of the backscattered Stokes $E_{2}$ pulse).

The spectra are depicted in Figs. 6 and 7. The backward spectrum, obtained by using the experimental setup of Fig. 4, exhibits as many as five Stokes lines (Fig. 6), and the forward spectrum (Fig. 7, corresponding to the experimental setup of Fig. 5) as many as five Stokes lines and four antiStokes lines. The frequency shift between two adjacent lines corresponds to the Brillouin spectral shift $\left(\nu_{s}=34\right.$ $\mathrm{GHz}$ ), and the number of spectral lines increases with the coupled pump power level.

The experiment could be improved by analyzing the Brillouin spectrum with a spectrum analyzer, which would permit better resolution of the fine structure of the Brillouin line and better interpretation of the process that leads to pulse generation.

\section{Discussion}

Starting from some experimental observations, a simple explanation for the frequency-multiplication rate may be given, although details of exactly what happens in the oscillator need not be known. We have to assume only (1) oscillator quasi-stationarity, which corresponds to the experimental observation in a time range of the order of $100 \mu \mathrm{sec}$, (2) exponential gain (which is valid for the low pump levels used here), and (3) strong enough pump depletion to reduce the pump level below the Brillouin stimulation threshold after the interaction with a backscattered Brillouin pulse (which

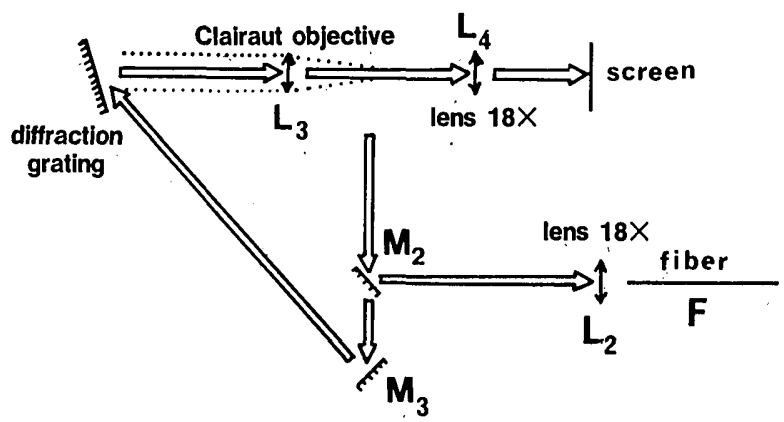

Fig. 4. Mounting set for the observation of the backward power spectrum corresponding to the setup of Fig. 1.

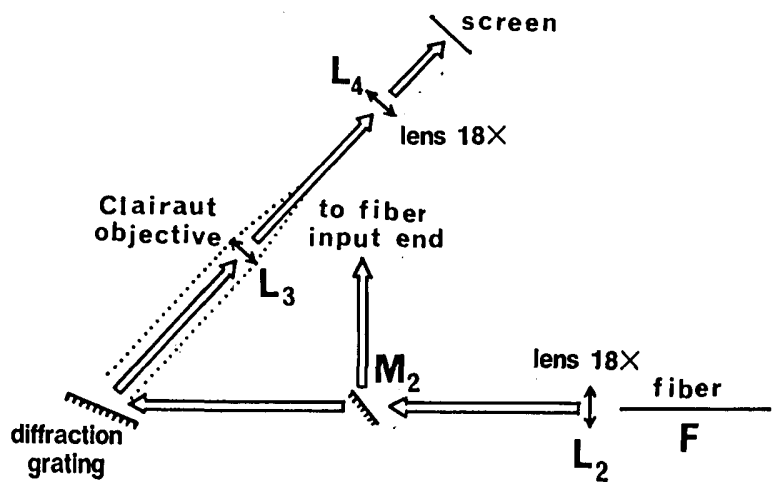

Fig. 5. Mounting set for the observation of the forward power spectrum corresponding to the setup of Fig. 1 . 


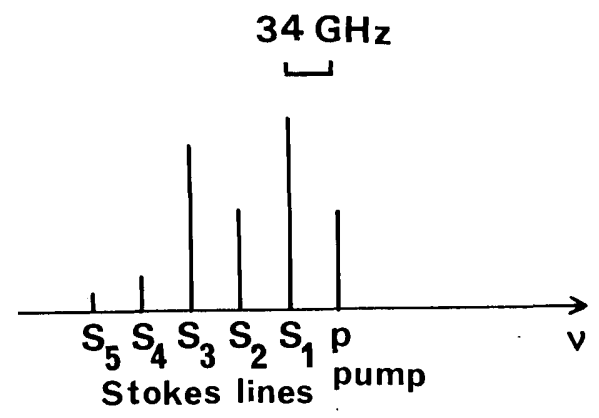

Fig. 6. Schematic diagram of the backward power spectrum at a high pumping level (the length of each line is proportional to its intensity).

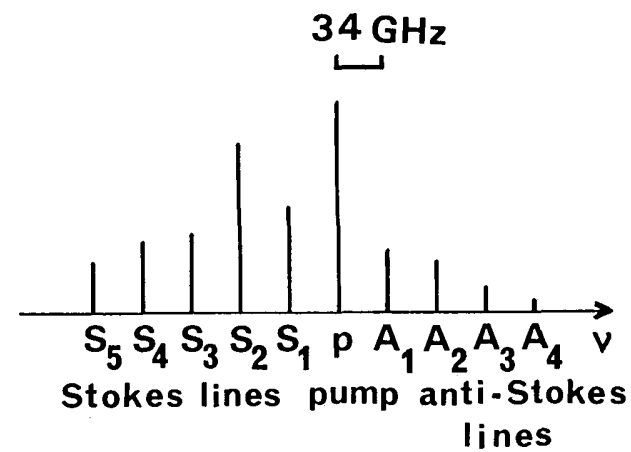

Fig. 7. Schematic diagram of the forward power spectrum at a high pumping level (the length of each line is proportional to its intensity).

corroborates the experimental observation of the pump transmission saturation).

Then if $T$ is the ring linear transmission coefficient for the Brillouin pulses during one round trip, $P$ the pump power coupled into the fiber, $G$ the gain Brillouin coefficient in the proper units, and $X$ an effective interaction length taking into account pump-wave attenuation and depletion along the fiber, stationarity implies equality between gain and losses, i.e., the product of $T$ times the Brillouin peak power in the ring just at the end of amplification equals the Brillouin peak power just before its interaction with the pump wave:

$$
T \exp (G P X)=1 \leftrightarrow X=-\ln (T) /(G P) .
$$

The interaction length along the fiber is then determined by the pump power level that results in an equal spacing between adjacent Brillouin pulses inside the ring.

Now, assuming $N$ Brillouin pulses traveling together in the ring, the leading pulse in the fiber begins to interact with the pump at a distance $X_{1}$ from the pump input fiber end. If $L$ is the ring optical length, we have at this particular time

$$
X_{1}=L-X_{N}
$$

where $X_{N}$ is the $N$ th pulse position at the same time. On the other hand, the shortest distance between the two successive pulses $N$ and 1 in the ring is

$$
\Delta_{N 1}=L-X_{N}+X_{1}
$$

from which we may deduce that
$X_{N}=L-X_{1}=L+X_{1}-\Delta_{N 1} \leftrightarrow \Delta_{N 1}=2 X_{1}=-2 \ln (T) /(G P)$,

which yields

$$
\begin{gathered}
\Delta_{12}+\Delta_{23}+\ldots+\Delta_{N 1}=N \Delta_{12}=L=-2 N \ln (T) /(G P) \\
\leftrightarrow \\
N=-G P L /[2 \ln (T)] .
\end{gathered}
$$

The number of pulses traveling together inside the cavity is then proportional to the coupled pump power level, and therefore the system will be stable for the particular pump power levels that ensure that $N$ is an integer. If $P$ is not equal to one of these particular values, stationarity is not possible; actually, what is experimentally observed is that there is a part of the Brillouin backscattering that is stationary; the recurrence frequency of that part increases by jumps when the power level is growing.

From the equation immediately above, we may deduce that

$$
P=-2 N \ln (T) /(G L),
$$

which permits calculation of the critical power corresponding to the fundamental repetition rate (corresponding to $N=1)$ :

$$
\begin{aligned}
& T=(0.5)^{2} \text { (the two beam splitters) } \times 0.8 \text { (the coupling } \\
& G=\text { (the silica Brillouin gain coefficient: } g=4.6 \\
& L=83 \mathrm{~m} \\
& P=-2 \log (0.11) /(6.4 \times 83) W=8 \mathrm{~mW} .
\end{aligned}
$$

This averaged pump power coupled into the fiber corresponds roughly to $30 \mathrm{~mW}$ at the argon-laser output, which is in good agreement with the experiment (less than $50 \mathrm{~mW}$, limited by the measurement precision).

A feature that does not correspond in this experiment to the standard multiple Brillouin scattering model is the existence of a forward scattering that appears in the spectrum. With high enough pump power level, the first (backward) Stokes component, downshifted by $\nu_{s}$ from the pump line frequency, may induce a second (forward) Stokes component, downshifted by $2 \nu_{s}$ from the pump frequency, which may generate a third (backward) Stokes at $3 v_{s}$, and so on. But the experimental observation exhibits a spectral shift of only $\nu_{s}$ instead of $2 \nu_{s}$ between adjacent lines in the backward spectrum (Fig. 6) as well as in the forward spectrum (Fig. 7). Moreover, this last spectrum shows an anti-Stokes wing not allowed in a three-wave Brillouin process. Nevertheless it is interesting to note that on the backward spectrum (Fig. 6) the third Stokes line amplitude is higher than the second one and that on the forward spectrum the second Stokes amplitude is higher than the first one, which indicates that the cascade process described above probably happens, but not alone.

This experimental feature may be explained by the fact that the pump laser is not isolated from the ring. $\mathrm{M}_{1}$ is a $50 \%$ beam splitter; half of the backward Brillouin pulse energy leaving the fiber through $L_{1}$ comes backward to the pump 
laser. A small part of these backward pulses is transmitted through the argon-laser front mirror inside the pump laser cavity and may perturbate the pump laser itself slightly, but the largest part is reflected from the laser front mirror and comes back forward into the ring oscillator. Three waves are then to be considered inside the ring oscillator: the forward pump wave $(p)$, the backward Brillouin pulses $\left(B_{1}^{-}\right)$, and also the forward Brillouin pulses $\left(B_{1}{ }^{+}\right)$. This configuration leads to the generation of several other waves: at first, by Brillouin processes, pulses $B_{1}$ will generate new forward and backward Brillouin pulses $\left(B_{2}\right)$, which by the same process may generate higher-order Brillouin pulses. Moreover there will be possibilities of constructive fourwave mixing among all these frequencies along the forward direction: for example, $\left(p+B_{1}{ }^{+}\right)$may generate $\left(B_{2}{ }^{+}+A_{1}{ }^{+}\right)$, where $A_{1}{ }^{+}$is an anti-Stokes component upshifted by $\nu_{s}$ from the pump line frequency. A similar scenario has been described in Refs. 28 and 30.

\section{B. Second Ring Oscillator}

The ring described above encounters several problems, among them (1) a complication of the wave configuration by several forward and backward Brillouin pulses, which interact with one another; (2) a coupling of the pump laser cavity with the Brillouin oscillator, which may perturbate the pump wave; and (3) a cw pumping regime that prevents spontaneous phonon damping after interaction with a particular Brillouin pulse. An acoustic phonon velocity of $c_{s}=$ $5960 \mathrm{~m} / \mathrm{sec}$ corresponds to a displacement of roughly $2.5 \mathrm{~mm}$ during one round-trip time of the Brillouin pulses, i.e., phonons may be considered motionless between two successive Brillouin pulse round trips. This phonon population, generated during the interaction between the pump wave and the Stokes peak, continues to be stimulated by the partially depleted cw pump beam and the Stokes tail and cannot be spontaneously damped. This process induces a spreading of the Brillouin pulse tail, a stronger pump depletion at the entry of the fiber, and therefore a shortening of the pump interaction length with the next regular-feedback Brillouin pulse. The spontaneous damping rate of the acoustical phonons is $\gamma_{s}=\pi \Delta \nu_{B}$, corresponding to a characteristic time $t_{s}$ $\simeq 6$ nsec, i.e., much shorter than the photon round-trip time $t_{r}=415 \mathrm{nsec}$. We find it useful to interrupt the pump a time interval longer than $t_{s}$ and shorter than $t_{r}$ in order to remove the remaining postinteractive phonons from the fiber.

To stabilize the Brillouin pulsed regime, we introduce an intracavity AOM. The AOM works in first-order Bragg diffraction with an efficiency of $70 \%$ (Fig. 2). $\mathrm{M}_{1}$ and $\mathrm{M}_{2}$ are now two $100 \%$ mirrors, which improve the Brillouin feedback coefficient (note that the pump feedback coefficient is therefore also increased). Observation of Brillouin pulses and output pump beam is carried out by reflection on glass beam splitters $S_{1}$ and $S_{2}$. The AOM is externally activated with a frequency $\bar{\nu}_{r}=2.427 \mathrm{MHz}$, this value being critical for the experimental pump threshold level. Note that $\bar{\nu}_{r}$ corresponds to a time of $412 \mathrm{nsec}$, slightly lower than the experimental Brillouin pulse round-trip time of $415 \mathrm{nsec}$, but this discrepancy may be attributed to experimental uncertainty.

The pump beam coupled into the fiber by the first Bragg order of the AOM generates backward Brillouin pulscs. They are maintained and self-synchronized in the ring cavity through the zero order of the AOM (when it is not activat- ed). The result is a better feedback rate in the cavity and also the isolation of the pump argon laser. The pump level in the fiber is not continuous but varies periodically at frequency $\vec{\nu}_{r}$, which ensures that the time interval for the stimulated phonons is spontaneously damped. In fact, at so large a modulation frequency the $\mathrm{AOM}$ has a sine response: during interaction the pump level cannot be considered constant, and the pump laser isolation is not complete. Nevertheless, we have not seen any evidence of forward Brillouin pulses in this ring oscillator, even at high pump power level.

In this configuration the ring behavior is much simpler than in the first one: the power spectral analysis shows one backward Brillouin Stokes line, and at a pump power level

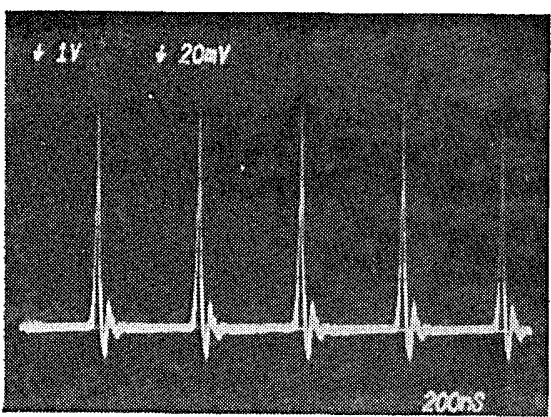

Fig. 8. Temporal structure of the backward-stimulated Brillouin beam during ring oscillation showing a typical train of stimulated Brillouin pulses in the short-time range (200 nsec/division) where the emission is highly stable.

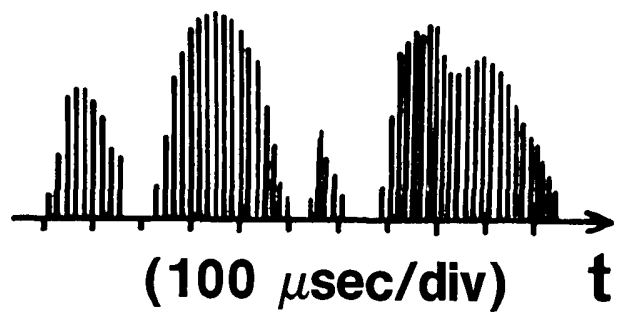

\section{low pump power}

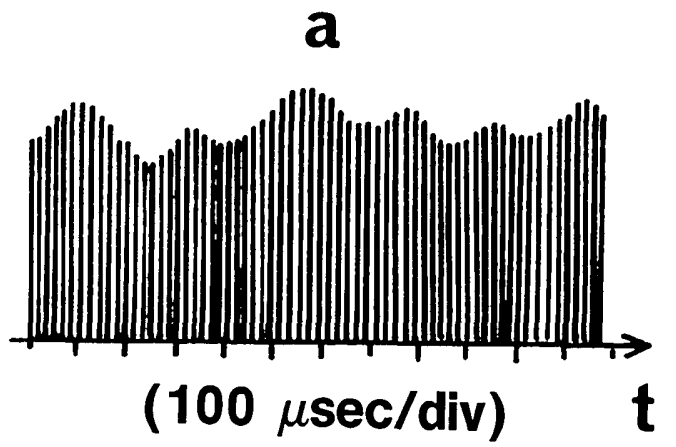

high pump power

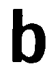

Fig. 9. Long-time envelope scheme of the stimulated Brillouin pulses at $a$, low and $b$, high pump power levels. 


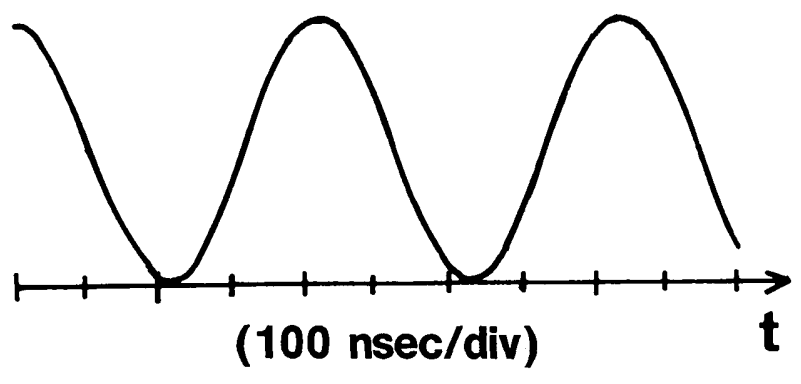

nondepleted pump

a

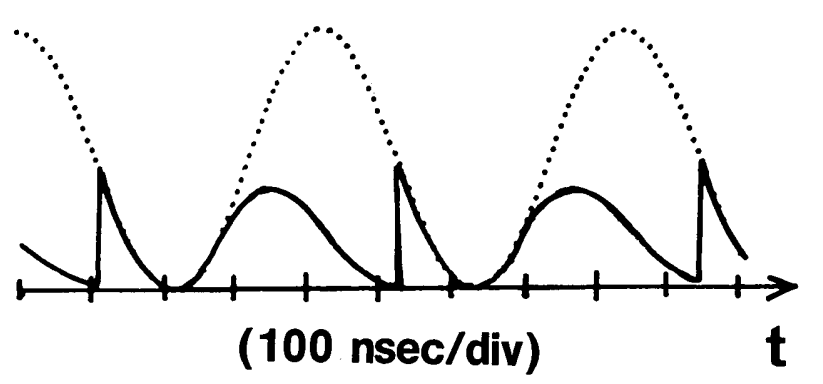

depleted pump

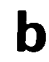

Fig. 10. Temporal structure of the forward-transmitted pump beam: a, without and $b$, with ring oscillation.

low enough (experimentally lower than $400 \mathrm{~mW}$ at the output of the argon laser) to prevent the fiber from oscillating by its end faces the repetition-rate frequency is well stabilized (Fig. 8).

Nevertheless, the peak amplitude of the Brillouin pulses remains unstable, particularly near the pump threshold (now $\sim 180 \mathrm{~mW}$ for the argon-laser power, much higher than for the first ring oscillator): when the observation is achieved within a long time scale (100 $\mu$ sec/division), the Brillouin output exhibits randomly distributed trains of periodic pulses. Higher power results in a partial stabilization, with amplitude fluctuation limited to some $20 \%$. This feature is shown schematically in Fig. 9. The mechanical vibrations could be partially responsible for these instabilities, because we already observe small amplitude fluctuations with similar frequencies below the Brillouin threshold; we attribute these instabilities to phase fluctuations between direct and recoupled pump beams.

The Brillouin pulse width is typically $20 \mathrm{nsec}$ FWHM, with incidentally shorter pulses down to $10 \mathrm{nsec}$ FWHM for the strongest amplitudes, and that width covers all the useful pump power range (roughly a factor of 2 , from 200 to 400 $\mathrm{mW}$ at the argon-laser output). The SBS amplification is strong enough to produce a Brillouin Stokes peak power as much as an order of magnitude higher than the nondepleted pump power (measured by opening the ring cavity).

Another interesting feature is the pump power depletion, shown in Fig. 10 for the case of high pumping level. Pump depletion is increasing with the power value and may reach more than $50 \%$ of the average level and $85 \%$ of local level.

\section{NUMERICAL RESULTS}

SBS in an optical-fiber ring configuration is one of the most striking examples of long-time nonlinear interaction of light with matter, and the dynamical evolution requires long-time numerical integration for the asymptotic behavior to be known. It may be modeled well by the one-dimensional C3W-SBS Eqs. (5)-(7) or (11), (12), and (7) when the optical Kerr effect is included. We have performed several numerical computations for the periodical feedback conditions of the ring configuration and for different pump powers $P$ given in Table 1 by using the four-step Runge-Kutta integration algorithm described in Ref. 16. Even though our principal result concerns the stabilization of a pump-modulated fiber-ring SBS laser, we present below some results concerning the $\mathrm{cw}$ fiber-ring configuration to show the lack of performance of such a setup for obtaining a stable SBS pulsed laser, as mentioned in Section 1 .

\section{A. Constant Pump Input (Continuous Wave)}

As we mentioned in Subsection 2.B, the SDA model, which neglects the inertial response time of the phonons, cannot describe the transient regime of amplification and compression of an initial bounded condition for $E_{2}(x, t=0)$ since its tail is spread because of the finite response time of the phonons. Therefore we use the C3W-SBS equations to describe the transient temporal behavior. Nevertheless, as we shall see, when the $E_{2}(x, t)$ envelope is spread through the entire fiber length, the pump depletion leads to a shortening of the gain medium, the Stokes peak gives power back to its tail, and the amplitude of the Stokes pulses decreases to a level low enough that the regime of decreasing relaxation oscillations tending to a steady state can be roughly described by the SDA model.

The high-amplitude transient regime depends on the initial conditions; in particular, the maximum amplitude of the amplified and compressed Stokes wave envelope depends on the amplitude and width of the initial Stokes noise or phonon noise. However, starting with a large variety of initial conditions, we have obtained the same asymptotic quasisteady state of decreasing relaxation oscillations for the Stokes pulses; this state depends only on the gain length $L / \Lambda$ (see Table 1) and on the feedback efficiency $\rho$, which takes into account the losses caused by the beam splitters and the coupling-recoupling efficiencies [here $\rho=0.16=(0.5)^{2}$ (the two beam splitters) $\times 0.8$ (the coupling efficiency) $\times 0.8$ (the recoupling efficiency)].

In Figs. 11-13 we show the numerical results of integration of Eqs. (5)-(7) for the ring configuration with constant pump input (cw) for the initial conditions $E_{s}(x, t=0)=0$ and a bounded Stokes bump of small amplitude $\left|E_{2}(x, t=0)\right|_{\max }=$ $10^{-6}$, which occupies half of the fiber length, in order to show a strong transient peak compression that is due to the SBS effect. In the optimal situation in which the backscattered Stokes pulses are isolated from the output pump mirror (e.g., through a Faraday rotator) in order to prevent backreflection into the fiber, the boundary conditions are

$$
E_{1}(0, t)=E_{p}+\sqrt{\rho} E_{1}(L, t), \quad E_{2}(L, t)=\sqrt{\rho} E_{2}(0, t),
$$

where $E_{p}$ is the pump field amplitude coupled into the fiber and $\rho$ is the feedback efficiency. The phase dynamics does not affect the SBS gain within the model described by Eqs. 

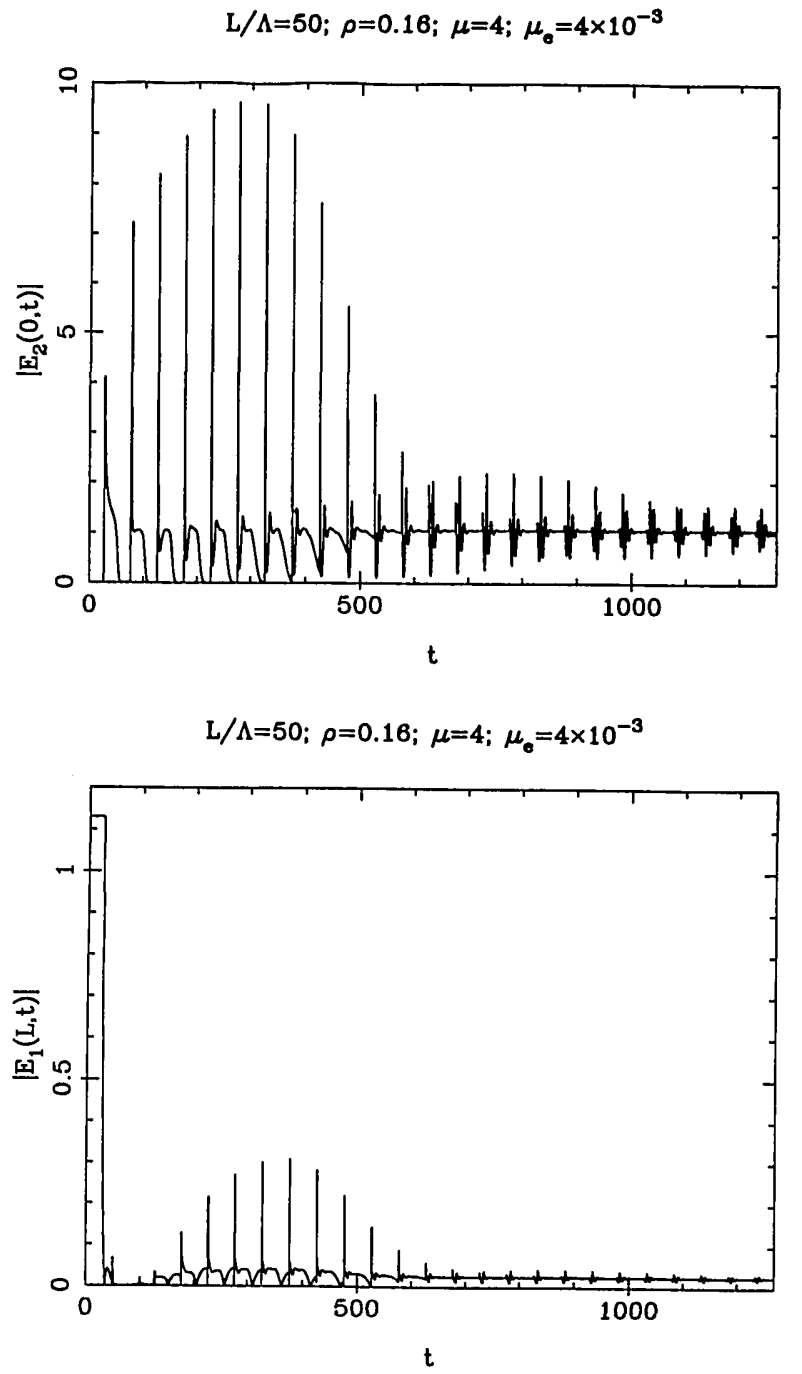

Fig. 11. Numerical computation of the pure C3W-SBS problem governed by Eqs. (5)-(7) for constant pump input $P=50 \mathrm{~mW}$ (and corresponding parameters of the second column of Table 1), and an initial bump condition for the Stokes background of amplitude $\left|E_{2}(x, 0)\right|_{\max }=10^{-6}$ and width $L / 2$. Time evolution for the amplitudes a, of the backscattered train of Stokes pulses $\left|E_{2}(x=0, t)\right| ; \mathrm{b}$, of the transmitted pump $\left|E_{1}(x=L, t)\right|$, which initially exhibits strong depletion and for long times reaches a quasi-constant small level.

(5)-(7). The pump input power coupled into the fiber is approximately $P=50 \mathrm{~mW}$, corresponding to $L / \Lambda=50$ and $\mu$ $\simeq 4$ (see Table 1 ). We can see a transient regime of strong amplified and compressed pulses (see Fig. 11a), the amplitude saturation of which depends on the width of the initial bump condition, accompanied by strong depletion of the pump (Fig. 11b). Figure 12 shows the envelope spatial distribution of the field amplitudes $\left|E_{i}(x, t)\right|$ in the fiber at consecutive time fractions of one round-trip period $\tau_{r}=50$ during the transient amplification regime. The leading backscattered Stokes pulse periodically encounters the fresh pump envelope near the middle of the fiber and exhibits noticeable gain close to the fiber input end. As we said above, for long times the inertial response of the excited phonons (finite response time) is responsible for their accumulation through the fiber medium, principally at the pump input end, and saturates the Stokes gain by lengthening the backscattered envelope until the Stokes pulses overlap (Fig. 11a). Then the pump monotonically exhibits depletion from the input end of the fiber, and the dynamics evolves to a regime of decreasing relaxation oscillations with an increasing of modulation frequency (Fig. 13). The result is an almost total reflector, which downshifts the pump frequency by the $34-\mathrm{GHz}$ sound frequency but avoids a SBS pulsed regime.

For the input pump powers used here, the SDA model described in Ref. 12 for $\gamma_{e}=0$ yields a steady state. The

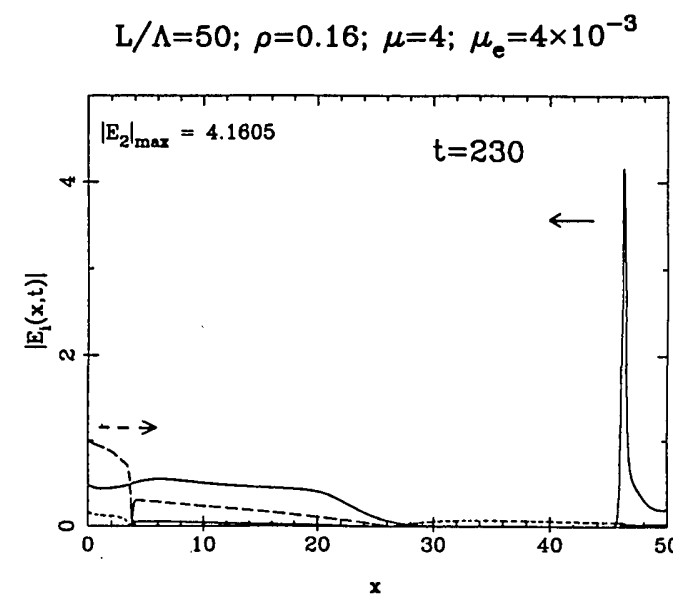

a

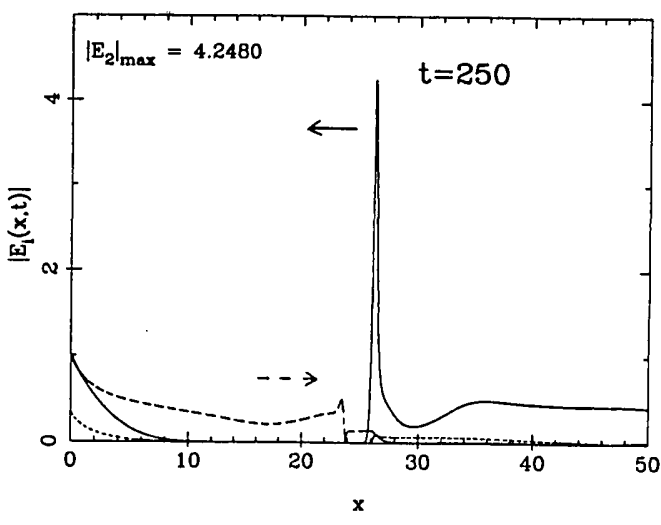

b

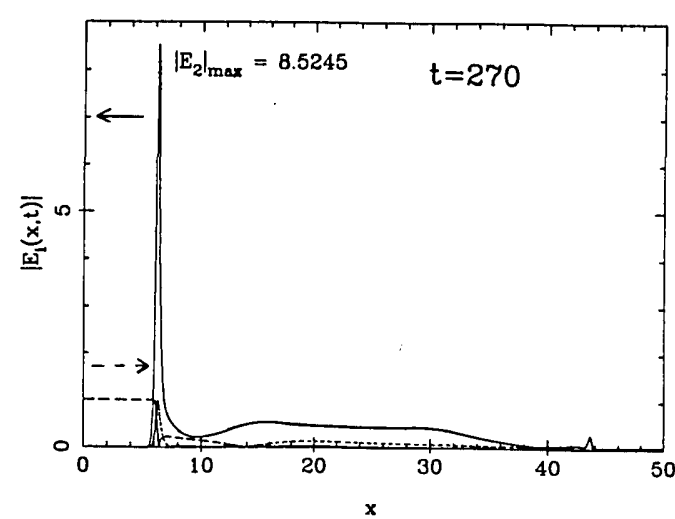

Fig. 12. Numerical computation for the C3W-SBS ring with constant pump input and the same parameters as for Fig. 1. Spatial distribution of the field envelope amplitudes in the fiber: $\left|E_{1}(x, t)\right|$ (long-dashed curves), $\left|E_{2}(x, t)\right|$ (solid curves), and $\left|E_{s}(x, t)\right|$ (shortdashed curves) at consecutive time intervals during one round-trip period $\tau_{r}=50$ in the transient regime of Fig. 11a. a, $t=230 ; \mathrm{b}, t=$ $250 ; \mathrm{c}, t=270$. 
$\mathrm{L} / \Lambda=50 ; \rho=0.16 ; \mu=4 ; \mu_{0}=4 \times 10^{-3}$
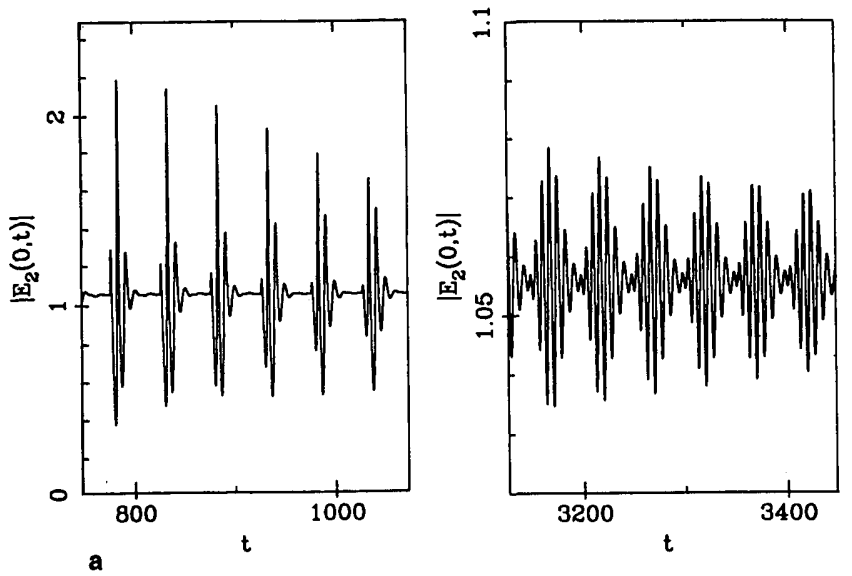

$\mathrm{L} / \Lambda=50 ; \rho=0.16 ; \mu=4 ; \mu_{\mathrm{e}}=4 \times 10^{-9}$

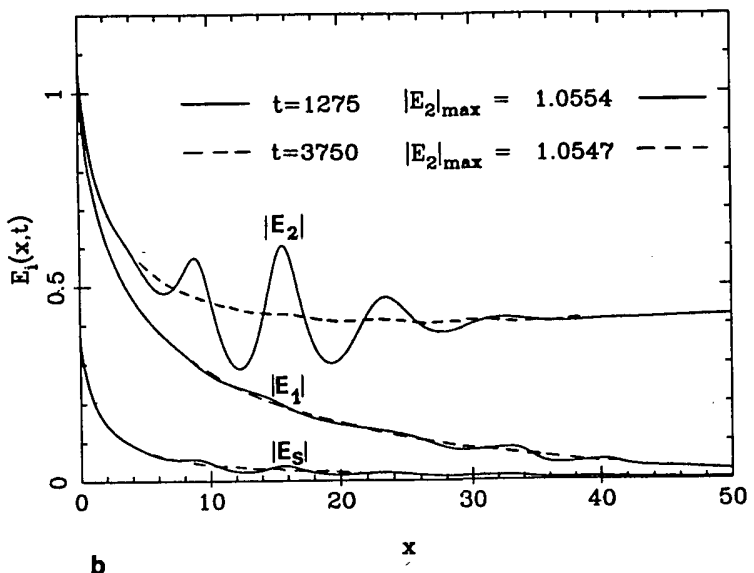

Fig. 13. a, Magnification of part of Fig. 11a at the same time interval. The maximum Stokes amplitude is of order 1 (in $E_{p}$ units) at $t \sim 1000$ and at this time presents four oscillations inside one period; it is of order 0.03 (in $E_{p}$ units) at $t \sim 3400$ and at this time presents seven oscillations inside one period. $b$, Spatial distribution of the field envelope amplitudes $\left|E_{i}(x, t)\right|$ in the fiber at time $t=$ 1275 (solid curves) and at time $t=3750$ (dashed curves), where the spatial envelope amplitudes tend to a monotonic quasi-steady distribution.

boundary conditions for this first ring configuration described by Eqs. (8) and (9) are given by

$$
\Phi_{1}(0, t)=\Phi_{\text {in }}+\rho \Phi_{1}(L, t), \quad \Phi_{2}(L, t)=\rho \Phi_{2}(0, t),
$$

where $\Phi_{\text {in }}$ is the pump flux power coupled into the fiber and $\rho$ is the feedback efficiency. For $\gamma_{e}=0$, it is shown in Ref. 12 that a steady-state solution exists:

$$
\begin{aligned}
& \frac{\Phi_{2}(0)}{\Phi_{1}(0)}=\frac{1-\rho \exp \left\{g L \Phi_{1}(0)\left[1-\Phi_{2}(0) / \Phi_{1}(0)\right]\right\}}{1-\rho}, \\
& \Phi_{1}(0)=\frac{\Phi_{\text {in }}}{1-\rho^{2} \exp \left\{g L \Phi_{1}(0)\left[1-\Phi_{2}(0) / \Phi_{1}(0)\right]\right\}} .
\end{aligned}
$$

We can see from Fig. 13 that an almost steady state is approached only for long times for $\gamma_{e}=0$ as well as for $\gamma_{e} \neq$ 0 . We can see in Fig. $11 \mathrm{~b}$ the transmitted pump $\left|E_{1}(L, t)\right|$, which is completely depleted at the beginning and increases with time until it reaches an almost constant small value.

In the strong-amplification regime the optical Kerr effect may become important, and in Fig. 14 we plot the numerical results of integration of Eqs. (11), (12), and (7) for the previous data and for $K_{r}=6 \times 10^{-3}$. We see that self-amplitude modulation saturates the Stokes-pulse amplitude $\left|E_{2}(x, t)\right|$ at lower values (compare it for $t=270$ with that correspond-
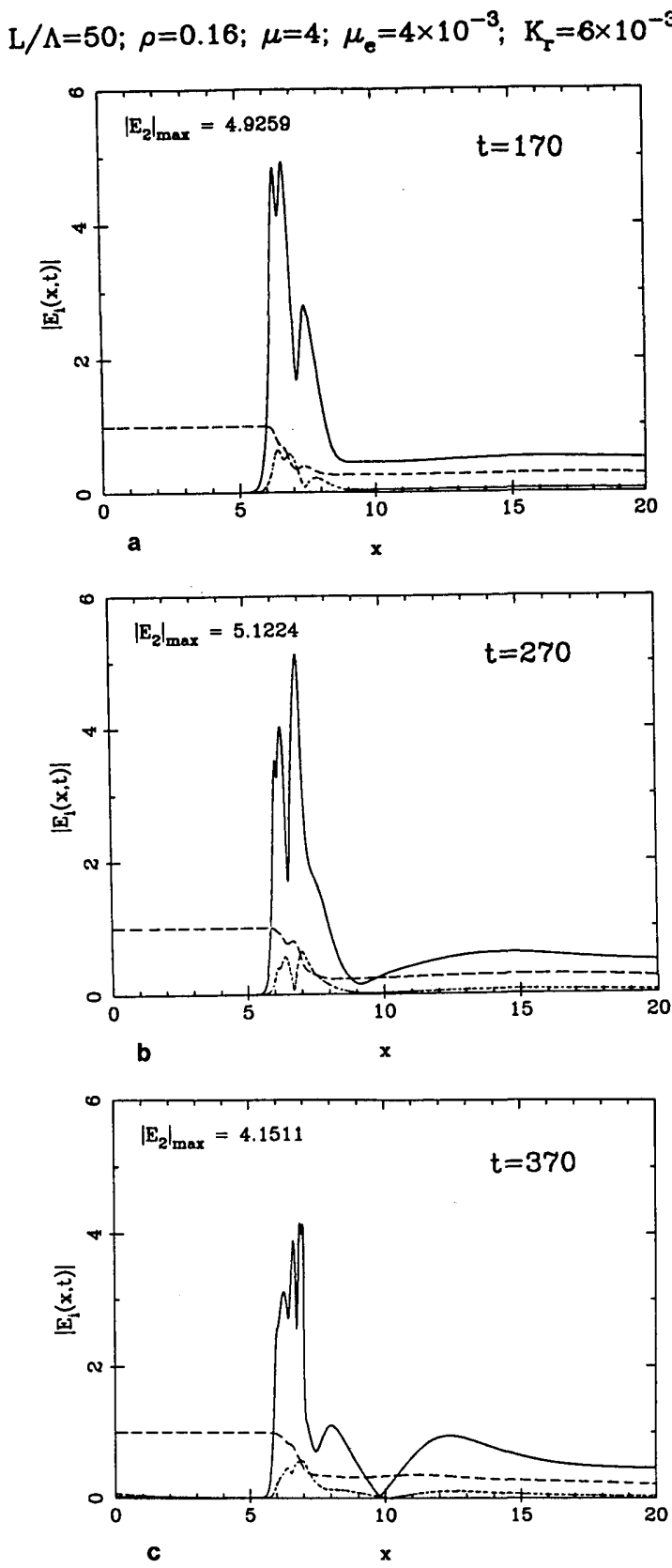

Fig. 14. Numerical computation for the C3W-SBS-Kerr ring with constant pump input, taking into account the optical Kerr effect [Eqs. (11), (12), and (7)]. $K_{r}=6 \times 10^{-3}$, and the other parameters correspond to those in the second column of Table 1. Spatial distribution of the field envelope amplitudes in the fiber: $\left|E_{1}(x, t)\right|$ (long-dashed curves), $\left|E_{2}(x, t)\right|$ (solid curves), and $\left|E_{s}(x, t)\right|$ (shortdashed curves) at three times separated by two round-trip periods $\tau_{r}$ $=50$ around the Stokes pulse amplitude saturation. The Stokes envelopes show a fine structure. The figures represent the first two fifths of the fiber. 
$\mathrm{L} / \Lambda=40 ; \rho=0.56 ; \mu=5 ; \mu_{0}=5 \times 10^{-3} ; \mathrm{K}_{\mathrm{r}}=0$

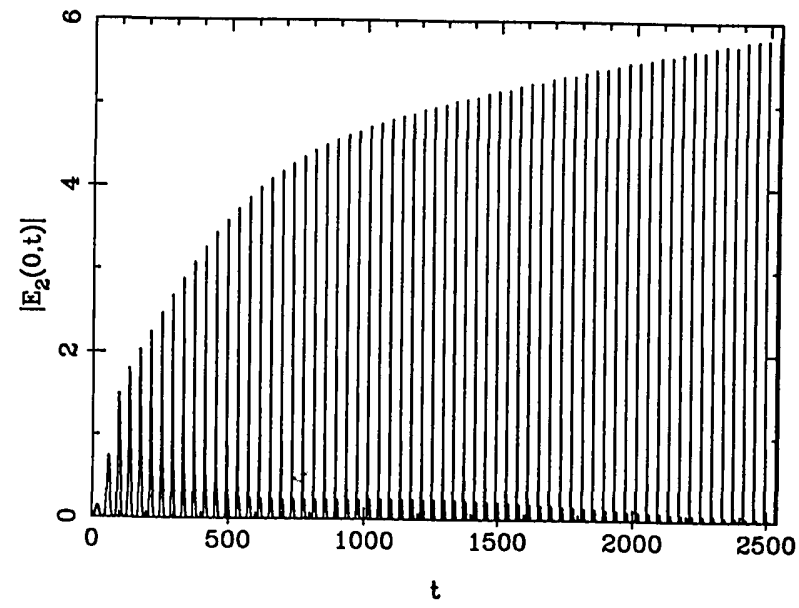

Fig. 15. Numerical computation for the SBS ring with modulated pump input (without the optical Kerr effect): time evolution for the amplitudes of the backscattered train of Stokes pulses $\mid E_{2}(x=0$, $t) \mid$, which show a slow secular amplification after time $t \sim 1000$. (Data from first column of Table 1 with $K_{r}=0$ ).

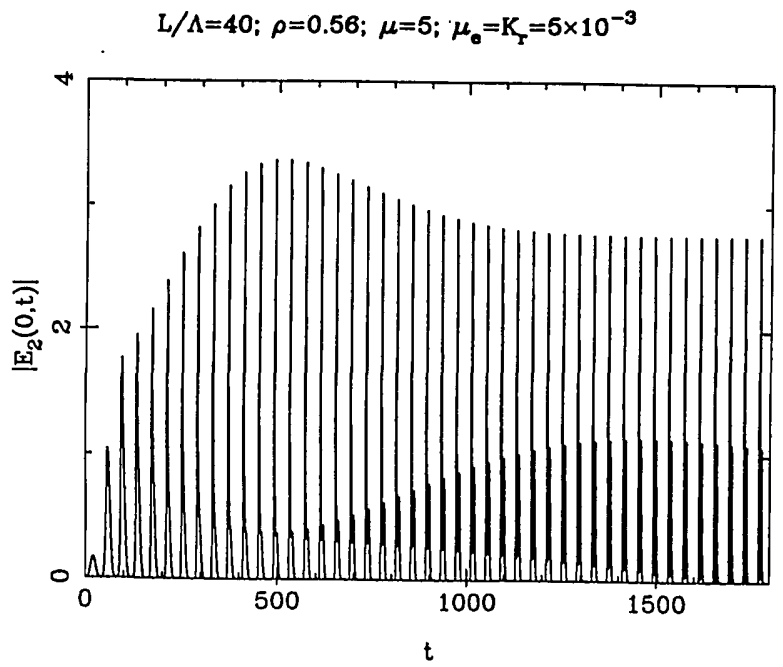

Fig. 16. Numerical computation for the SBS-Kerr ring with modulated (MW) pump input (with the optical Kerr effect and data corresponding to those of the first column of Table 1 with $K_{r}=5 \times$ $10^{-3}$ ): Time evolution for the amplitudes of the backscattered train of Stokes pulse $\left|E_{2}(x,=0, t)\right|$, which show a saturation at time $t \sim$ 500. The asymptotic stage presents an amplitude $\left|E_{2}(x, t)\right|_{\max } \simeq$ 2.8 .

ing to Fig. 12c), and the envelope shows a fine structure. Figure 14 represents the first two fifths of the fiber. The envelope structure looks like the experimental one shown in Fig. 3b, which certainly indicates that the multiple-scattering experimental situation maintains a highly transient regime, like that of Ref. 31. However, here we do not consider interaction between multiple backward and forward Brillouin Stokes pulses, which were present in the actual experiment owing to the lack of isolation of the pump laser from the ring, and we obtain a quasi-steady-state regime.

The understanding of the saturation and relaxation mechanism for the cw pump input case, described by the C3W. SBS equations, leads us to look for a SBS pulsed regime. In order to permit spontaneous damping of the accumulated phonons at each round trip (the third problem mentioned in Subsection 3.B), we must turn off the pump after the output of the backscattered Stokes pulse. Therefore we maintain the periodic regime of amplified and compressed Stokes pulses by imposing on the pump intensity the strong modulation synchronized at the Stokes round-trip frequency.

\section{B. Modulated Pump Input}

For a modulated wave, corresponding to the second ring oscillator described in Subsection 3.B, where substitution of mirrors for the splitters yields $\rho=0.56$ for the feedback coefficient, strong periodical variation of the pump input

$$
\mathrm{L} / \Lambda=40 ; \rho=0.56 ; \mu=5 ; \mu_{\mathrm{e}}=\mathrm{K}_{\mathrm{r}}=5 \times 10^{-3}
$$
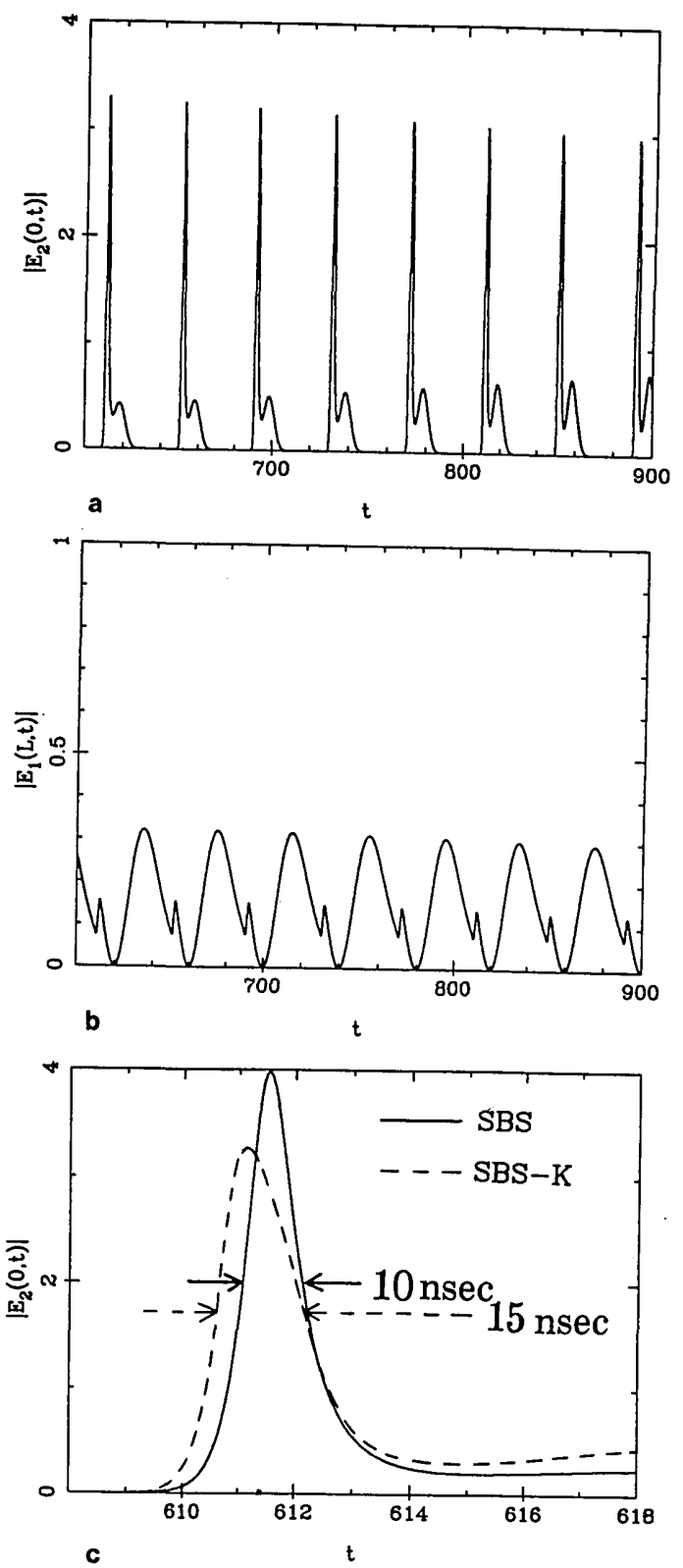

Fig. 17. a, Magnification of Fig. 16 showing several Stokes pulses $\left|E_{2}(0, t)\right|$. b, Several transmitted pump pulses $\left|E_{1}(L, t)\right|$ showing depletion. c, Comparative time width of the backscattered Stokes pulse corresponding to the SBS ring of Fig. 15 (solid curve) and of the SBS-K ring of Figs. 16 and 17 (dashed curve). 
$L / \Lambda=40 ; \rho=0.56 ; \mu=5 ; \mu_{e}=K_{r}=5 \times 10^{-3}$
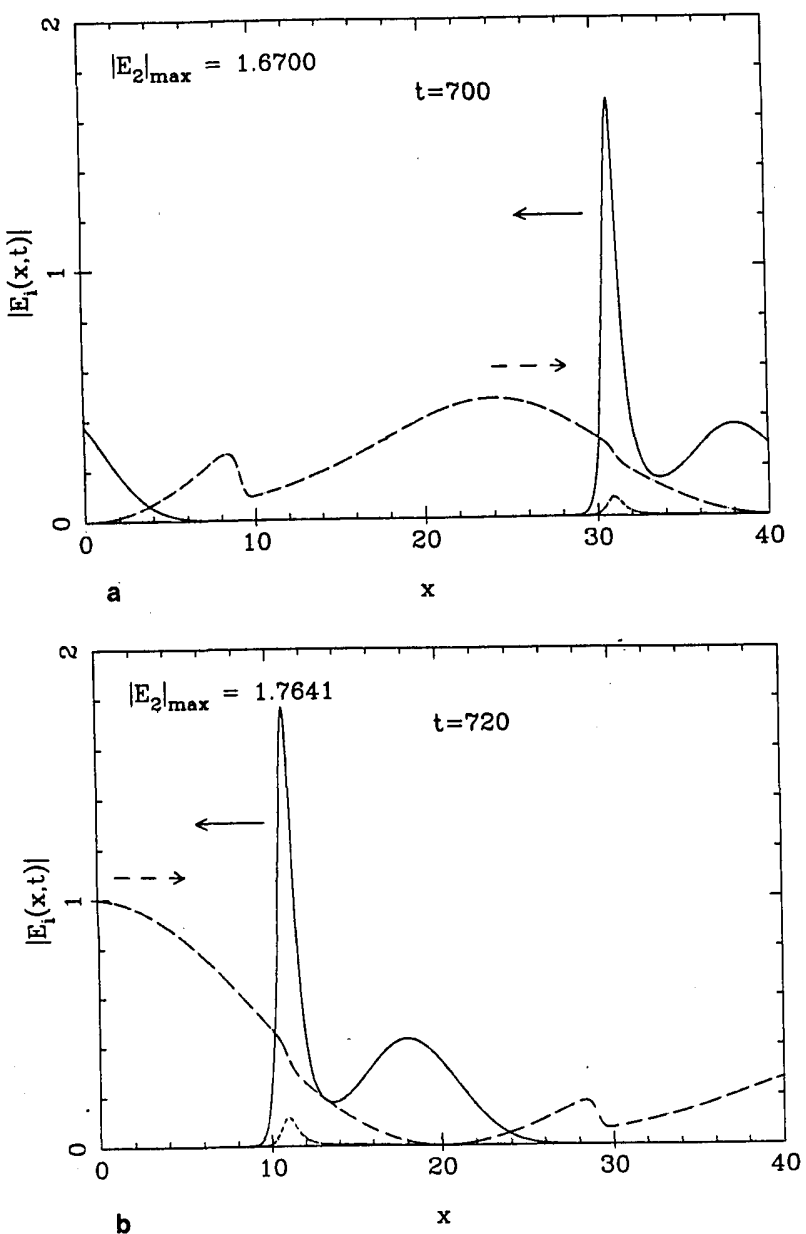

Fig. 18. Numerical computation for the SBS-Kerr ring with modulated pump input. Spatial distribution of the field envelope amplitudes in the fiber: $\left|E_{1}(x, t)\right|$ (long-dashed curves), $\left|E_{2}(x, t)\right|$ (solid curves), and $\left|E_{s}(x, t)\right|$ (short-dashed curves), at two consecutive times during one period.

level by the AOM at the round-trip frequency $\nu_{r}=1 / \tau_{r}$, synchronized with opposite transmission of the outgoing backscattered Brillouin pulses, determine the boundary conditions

$$
\begin{aligned}
E_{1}(0, t)= & E_{p}\left[1+\cos \left(2 \pi t / \tau_{r}\right)\right] / 2 \\
& +f \sqrt{\rho} E_{1}(L, t)\left[1-\cos \left(2 \pi t / \tau_{r}\right)\right] / 2, \\
E_{2}(L, t)= & \sqrt{\rho} E_{2}(0, t)\left[1-\cos \left(2 \pi t / \tau_{r}\right)\right] / 2
\end{aligned}
$$

that simulate the experimental situation. $f$ is a phase factor depending on the optical path, lying in the interval $[-1,+1]$. Here we arbitrarily take $f=1$. The numerical results are plotted in Figs. $15-18$ for $L / \Lambda=40, \mu=5$, and $\mu_{e}=5 \times 10^{-3}$, which represent a peak amplitude $E_{p}=1.4 \mathrm{MV} / \mathrm{m}$ or a peak power of approximately $P=25 \mathrm{~mW}$. Of course, the nonlinear SBS gain in the presence of the variable pump input is variable too, yielding a smaller mean power coupled into the fiber. This is taken into account in the computation of the C3W-SBS equations, for which all the dimensionless variables are parameterized to the peak amplitude $E_{p}$.

Figure 15 shows the train of backscattered Brillouin pulses obtained by integration of Eqs. (5)-(7). For long times the Stokes-pulse amplitude shows a small linear growth. Taking into account the optical Kerr effect described by Eqs. (11), (12), and (7), which result is plotted in Fig. 16, we obtain an almost stable saturated regime of backscattered Stokes pulses. Figure 17a is an enlargement of part of Fig. 16 containing several pulses in order to show the similarity with the experimental results and the stability of the system. The experimental behavior of the transmitted pump shown in Fig. 10b, which exhibits depletion, is in good quantitative agreement with that shown in Fig. 17b. The optical Kerr effect limits the Stokes-pulse compression, as is shown in Fig. 17c, and corresponds better to the experimental recording. Figure 18 shows the envelope spatial distribution of the field amplitudes $\left|E_{i}(x, t)\right|$ in the fiber at two consecutive times during one period of the stabilized pulsed regime.

\section{CONCLUSION}

A ring optical-fiber oscillator may be designed to produce, by SBS, a quasi-periodic train of compressed Stokes pulses amplified to several times $(\sim 10)$ the peak pump level. This result is obtained by modulating the pump wave coupling and the oscillator feedback by an AOM synchronized at the round-trip photon frequency. Such a device could be the basis of an all-optical-cw pumped clock if the modulator is driven by the Stokes pulse train itself.

In order to describe the dynamical evolution of this interaction properly, we have developed a numerical model based on the coherent partial differential equations for Brillouin scattering, which permits the optical Kerr effect to be taken into account and the limitations of the more classical intensity model to be known more precisely. Numerical and experimental results agree quantitatively. Moreover, this coherent model provides the possibility of generating quasisoliton Stokes pulses in a SBS fiber-ring laser. ${ }^{32}$

\section{ACKNOWLEDGMENTS}

The authors thank Pierre Sixou, Claude Froehly, and Henri Le Gall for use of the experimental facilities. They also thank Jean Coste and Olivier Legrand for stimulating discussions and Roger H. Stolen for providing the fiber used in these experiments and for helpful remarks. Part of this research has been developed in the scientific program of the Groupe d'Interaction Laser-Matière associated with the Laboratoire pour l'Utilisation des Lasers Intenses-Centre National de la Recherche Scientifique. The Laboratoire de Physique de la Matière Condensée is Laboratoire Unité de Recherche Associée 190, associated with the Centre National de la Recherche Scientifique, France. C. Montes is also with the Observatoire de Nice, Boite Postale No. 139, 06003 Nice Cédex, France.

\section{REFERENCES}

1. E. P. Ippen and R. H. Stolen, "Stimulated Brillouin scattering in optical fibers," Appl. Phys. Lett. 21, 539 (1972).

2. D. Cotter, "Stimulated Brillouin scattering in monomode optical fiber," J. Opt. Commun. 4, 10 (1983).

3. K. O. Hill, B. S. Kawasaki, and D. C. Johnson, "Cw Brillouin laser," Appl. Phys. Lett. 28, 608 (1976). 
4. D. C. Johnson, K. O. Hill, and B. S. Kawasaki, "Brillouin optical-fiber ring oscillator design," Radio Sci. 12, 519 (1977).

5. B. S. Kawasaki, D. C. Johnson, Y. Fujii, and K. O. Hill, "Bandwidth-limited operation of a mode-locked Brillouin parametric oscillator," Appl. Phys. Lett. 32, 429 (1978).

6. D. R. Ponikvar and S. Ezekiel, "Stabilized single-frequency stimulated Brillouin fiber ring laser," Opt. Lett. 6, 398 (1981).

7. L. F. Stokes, M. Chodorow, and H. J. Shaw, "All-fiber stimulated Brillouin ring laser with submilliwatt pump threshold," Opt. Lett. 7, 509 (1982).

8. A. Ya. Karasik and A. V. Luchnikov, "Generation of nanosecond radiation pulses as a result of stimulated Brillouin scattering in single-mode fiberglass waveguide," Sov. J. Quantum Electron. 15,877 (1985).

9. I. Bar-Joseph, A. Dienes, A. A. Friesem, E. Lichtman, R. G. Waarts, and H. H. Yaffe, "Spontaneous mode locking of single and multi mode pumped SBS fiber lasers," Opt. Commun. 59, 296 (1986)

10. V. N. Lugovoy and V. N. Streltsov, "Stimulated Raman radiation and stimulated Mandel'stam-Brillouin radiation in a laser resonator," Opt. Acta 20, 165 (1973).

11. R. V. Johnson and J. H. Marburger, "Relaxation oscillations in stimulated Raman and Brillouin scattering," Phys. Rev. A 4, 1175 (1971).

12. I. Bar-Joseph, A. A. Friesem, E. Lichtman, and R. G. Waarts, "Steady and relaxation oscillations of stimulated Brillouin scattering in single-mode optical fibers," J. Opt. Soc. Am. B 2, 1606 (1985).

13. C. L. Tang, "Saturation and spectral characteristics of the Stokes emission in the stimulated Brillouin process," J. Appl. Phys. 37, 2945 (1966).

14. W. Kaiser and M. Maier, "Stimulated Mandelstam-Brillouin process," in Laser Handbook 2, T. F. Arecchi and F. O. SchulzDubois, eds. (North-Holland, Amsterdam, 1972), p. 1077.

15. I. L. Fabelinskii, "Stimulated molecular scattering of light," in Molecular Scattering of Light, I. L. Fabelinskii, ed. (Plenum, New York, 1968), pp. 483-532; "Stimulated MandelstamBrillouin process," in Nonlinear Optics, Vol. I of Quantum Electronics: A Treatise, H. Rabin and C. L. Tang, eds. (Academic, New York, 1975), pp. 363-418.

16. J. Coste and C. Montes, "Asymptotic evolution of stimulated Brillouin scattering: Implications for optical fibers," Phys. Rev. A 34, 3940 (1986).
17. C. Montes and R. Pellat, "Inertial response to nonstationary stimulated Brillouin backscattering: damage of optical and plasma fibers," Phys. Rev. A 36, 2976 (1987).

18. R. H. Stolen and C. Lin, "Self-phase-modulation in silica optical fibers," Phys. Rev. A 17, 1448 (1978).

19. D. T. Hon, "Pulse compression by stimulated Brillouin scattering," Opt. Lett. 5, 516 (1980).

20. F. Chu and C. Karney, "Solution of the three-wave resonant equations with one wave heavily damped," Phys. Fluids 20, 1728 (1977).

21. R. H. Stolen and J. E. Bjorkholm, "Parametric amplification and frequency conversion in optical fibers," IEEE J. Quantum Electron. QE-18, 1062 (1982).

22. G. Lamb, Jr., in Elements of Soliton Theory, G. Lamb, Jr., ed. (Wiley, New York, 1980), p. 153.

23. J. Pelous and R. Vacher, "Thermal Brillouin scattering measurements of the attenuation of longitudinal hypersounds in fused quartz," Solid State Commun. 16, 279 (1975).

24. D. Heiman, D. S. Hamilton, and R. W. Hellwarth, "Brillouin scattering measurements on optical glasses," Phys. Rev. B 19, 6583 (1979)

25. K. Tai, A. Hasegawa, and A. Tomita, "Observation of modulational instability in optical fibers," Phys. Rev. Lett. 56, 135 (1986).

26. M. J. Potasek and G. P. Agrawal, "Self-amplitude-modulation of optical pulses in nonlinear dispersive fibers," Phys. Rev. A 36, 3862 (1987)

27. Y. S. Silberberg and I. Bar-Joseph, "Optical instabilities in a nonlinear Kerr medium," J. Opt. Soc. Am. B 1, 662 (1984).

28. K. O. Hill, D. C. Johnson, and B. S. Kawasaki, "Cw generation of multiple Stokes and anti-Stokes Brillouin-shifted frequencies," Appl. Phys. Lett. 29, 185 (1976).

29. R. H. Stolen, AT\&T Bell Laboratories, Holmdel, New Jersey 07733 (personal communication).

30. P. Labudde, P. Anliker, and H. P. Weber, "Transmission of narrow band high power laser radiation through optical fibers," Opt. Commun. 32, 385 (1980).

31. C. Montes and J. Coste, "Optical turbulence in multiple stimulated Brillouin backscattering," Laser Part. Beams 5, 405 (1987).

32. O. Legrand and C. Montes, "Apparent superluminous quasisolitons in stimulated Brillouin backscattering," J. Phys. Coll. (Paris) (to be published). 